\title{
Graph-Based Semi-Supervised Learning for Natural Language Understanding
}

\author{
Zimeng Qiu $^{\dagger, \ddagger}$, Eunah Cho ${ }^{\ddagger}$, Xiaochun Ma ${ }^{\ddagger}$, William M. Campbell ${ }^{\ddagger}$ \\ ${ }^{\dagger}$ Electrical \& Computer Engineering Department, Carnegie Mellon University \\ $\ddagger$ Amazon Alexa AI \\ zimengq@andrew.cmu.edu, \{eunahch,mxiaochu, cmpw\}@amazon.com
}

\begin{abstract}
Semi-supervised learning is an efficient method to augment training data automatically from unlabeled data. Development of many natural language understanding (NLU) applications has a challenge where unlabeled data is relatively abundant while labeled data is rather limited.

In this work, we propose transductive graphbased semi-supervised learning models as well as their inductive variants for NLU. We evaluate the approach's applicability using publicly available NLU data and models. In order to find similar utterances and construct a graph, we use a paraphrase detection model. Results show that applying the inductive graph-based semi-supervised learning can improve the error rate of the NLU model by $5 \%$.
\end{abstract}

\section{Introduction}

Natural language understanding (NLU) technology is an important component for a dialog system and is commonly used in voice assistants (e.g., Amazon Alexa, Google Home, Siri). An NLU system takes recognized speech input and produces intent, domain, and slots for the utterance to support the user request (Tur and De Mori, 2011). For example, for a user request "turn off the lights in living room" the NLU system might generate domain Device, intent Light-Control, and slot values of "off" for OffTrigger and "living room" for Location.

It is crucial for an NLU system to be able to add further support and improve performance in an incremental manner. An efficient method for this is semi-supervised learning (SSL), especially when only small amount of labeled data is available. In contrast with supervised learning algorithms, SSL algorithms can improve their performance by leveraging information in unlabeled data. Some recent results (Laine and Aila, 2017; Miyato et al.,
2019; Tarvainen and Valpola, 2017) have shown that semi-supervised learning could reach performance of purely supervised learning in certain scenarios.

Currently, most NLU models rely on the utterance text and its annotation to learn domain, intent, and slots of the utterance. However, this does not scale to unlabeled data. In this work, we aim to find and represent the relationship between labeled and unlabeled data in a non-Euclidean space, a graph, for SSL. We show that graph-based SSL is a high-performant method which improves an NLU model by leveraging unlabeled data.

In order to represent the labeled and unlabeled data in a graph, we used a paraphrase detection model. Nodes and edges in the graph represent utterances and paraphrase relations respectively. Given the constructed graph, a transductive graph model was applied for node classification, which in our case is intent classification (IC) for each utterance. We used an NLU Slot Gated Model (SGM) (Goo et al., 2018) to obtain slot labels. Experiments on the SNIPS data set show that we can achieve $5 \%$ error reduction on the slot error rate.

The rest of the paper is structured as follows: Section 2 reviews work related to our approach. Section 3 describes the graph-based SSL methods we propose in this paper followed by a description of the paraphrase measures used to construct a graph. Section 5 describes the experimental setup. We share results and analysis in Section 6. Section 7 shows conclusions.

\section{Related Work}

Over the past few years, many deep learning approaches have been extended to NLU tasks-e.g., intent classification and slot filling (Liu and Lane, 2016). Manual annotation is costly. Thus, recent work has turned to SSL in order to achieve similar 


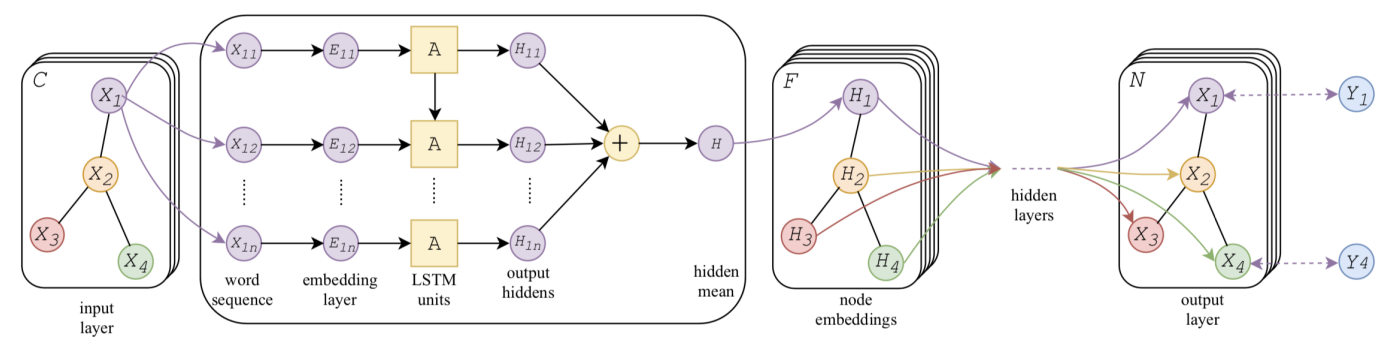

Figure 1: TGCN model architecture.

performance with much less manually annotated data compared to purely supervised learning.

Aliannejadi et al. (2017) applies graph-based supervised learning of Conditional Random Fields (CRF) for Spoken Language Understanding (SLU) on unaligned data.

Lan et al. (2018) proposes an adversarial multitask learning method by merging a bidirectional language model (BLM) and a slot tagging model (STM). As a secondary objective, the BLM is used to learn generalized and unsupervised knowledge with abundant unlabeled data and improve the performance of STM on unseen data samples.

Cho et al. (2019a) generates paraphrases and uses them to enhance the training set in a semisupervised learning setting for NLU. The augmented data is used jointly for domain classification, intent classification and slot filling.

The recent rise of neural networks has brought significant advances in a large number of machine learning tasks. While deep learning techniques have achieved huge success, their performance on non-Euclidean data is not as good as on Euclidean data. The complexity of graph structures is a significant challenge to most of existing deep learning algorithms and this complexity has drawn the attention of community to extend deep learning algorithms to graph data which in turn inspired various methods for Graph Neural Networks (GNN) (Kipf and Welling, 2017; Velickovic et al., 2018; Yang et al., 2018; Zhang et al., 2018a; Tran, 2018; Xinyi and Chen, 2019).

GNNs can be applied in a supervised, semisupervised, or purely unsupervised manner for different tasks. For instance, graph convolutional networks (GCN) (Kipf and Welling, 2017) could be used in a semi-supervised way for node-level classification (Kipf and Welling, 2017), in a supervised way for graph-level classification (Zhang et al., 2018b; Ying et al., 2018; Pan et al., 2016,
2017), and in an unsupervised way for graph embedding (Hamilton et al., 2017; Kipf and Welling, 2016; Pan et al., 2018; Yang et al., 2018).

To the best of our knowledge, our work is the first approach to apply a text-based graph structure for SSL for NLU. We evaluate our method on a publicly available data set in order to show its applicability.

\section{Graph Methods}

We propose two transductive graph models for semi-supervised learning NLU tasks, Text Graph Convolutional Network (TGCN) and Text Graph Beam Search (TeGrabS), as well as their inductive versions, Pseudo labeling with TGCN (PLTGCN) and Pseudo labeling with TeGrabS (PLTeGrabS).

\subsection{Transductive Models}

In a semi-supervised learning setting, we have the following data sets, $\mathcal{D}=$ $\left\{\mathrm{X}_{\text {train }}, \mathrm{X}_{\text {unlabeled }}, \mathrm{X}_{\text {test }}\right\} . \quad$ In inductive scenarios, labels of $\mathrm{X}_{\text {test }}$ and $\mathrm{X}_{\text {unlabeled }}$ are unknown to model, and the model sees $\mathbf{X}_{\text {unlabeled }}$ during training but $\mathrm{X}_{\text {test }}$ is unseen. In transductive scenarios, the model sees $\mathrm{X}_{\text {test }}$ and $\mathrm{X}_{\text {unlabeled }}$ at training time.

In our task, transductive models learn paraphrase patterns among utterances from a given graph, then they are applied as auxiliary models in the NLU model pseudo-labeling pipeline. By doing this, we make the determination of input utterances labels become a parametric function of the features and thus obtain inductive variants of transductive models.

\subsubsection{TeGrabS}

Similar to beam search, TeGrabS is a heuristic method. For a starting node $n$, the algorithm keeps track of $k$ separate transitions; for each transition 


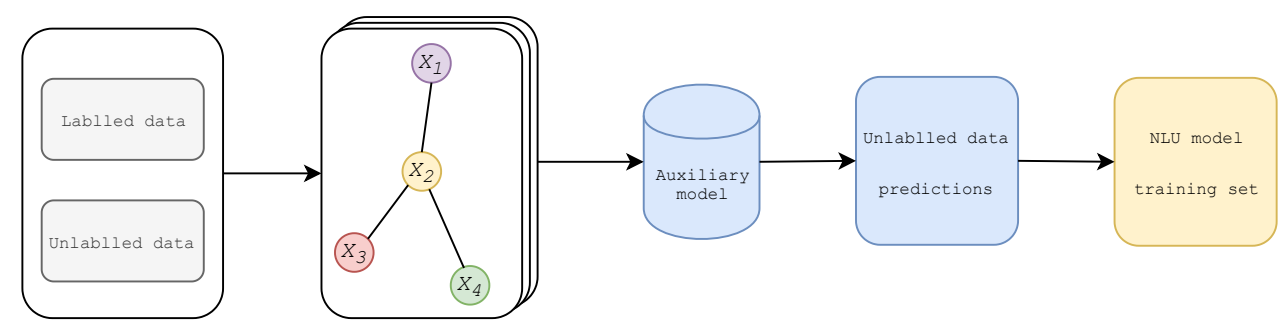

Figure 2: Inductive semi-supervised learning pipeline.

at each time step, random sample a node from the current node's neighbors as the next node. The sampling process can be regarded as a Markov chain: the transition is represented by hop from one node to another with the weight of the edge between two nodes is the transition probabilities.

$$
p\left(n^{\prime} \mid n\right)=W_{n, n^{\prime}}
$$

where $n^{\prime}$ is a candidate for next node, $W_{n, n^{\prime}}$ is weight of edge between $n$ and $n^{\prime}$. Probability of a whole transition is modelled as equation below:

$$
p\left(n_{0}, n_{1}, \cdots, n_{m}\right)=\prod_{i=0}^{m-1} W_{i, i+1}
$$

If a transition does not have available next node candidates (i.e., the current node does not have any neighbors), this transition will be stopped and the beam width is reduced by 1 . The beam search will be terminated when all transitions either meet the maximum number of hops limit or are stopped due to the current node not having any neighbors. Pseudocode of TeGrabS is shown as Algorithm ?? in the Appendix.

\subsubsection{TGCN}

GCN has been commonly applied on graph data in recent years (Kipf and Welling, 2017; Hamilton et al., 2017; Zhang et al., 2018b; Ying et al., 2018; Pan et al., 2016, 2017; Kipf and Welling, 2016; Pan et al., 2018; Yang et al., 2018). However, to the best of our knowledge, few researchers have attempted to use it for text graphs.

Here we propose our GCN-based transductive text-graph semi-supervised learning model, TGCN. The model architecture is shown in Figure 1. The input is a text graph including nodes $\left\{X_{1}, X_{2}, \cdots, X_{i}\right\}$ and edges where each node represents a unique utterance. We use an embedding layer followed by LSTM cells as a feature extractor; $X_{i j}, E_{i j}$ and $H_{i j}$ are token, word embedding, and LSTM hidden state for $j$-th word in $i$-th utterance, respectively.

$$
E_{i}=\frac{1}{n} \sum_{w \in X_{i}} E_{w}
$$

where $n$ is the length of utterance $X_{i}$. We compute the average sum of each token's hidden state in utterance as the node feature.

$$
H_{i}=\frac{1}{n} \sum_{j=0}^{n} H_{i j}
$$

Inspired by the original GCN architecture design (Kipf and Welling, 2017), the features are fed into a two-layer graph convolution network. The first graph convolution layer is followed by ReLU units,

$$
F^{(l+1)}=\sigma\left(\widetilde{D}^{-\frac{1}{2}} \widetilde{A} \widetilde{D}^{-\frac{1}{2}} F^{(l)} W^{(l)}\right)
$$

where $\widetilde{A}=A+I_{N}$ is the adjacency matrix of the undirected graph with self-connections added, $I_{N}$ is the identity matrix, $\widetilde{D}_{i i}=\sum_{j} \widetilde{A}_{i j}$ and $W^{(l)}$ is a layer-specific trainable weight matrix. $\sigma(\cdot)$ is an activation function, which is ReLU in our case. $F^{(l)}$ is the matrix of activations in $l$-th layer, $F^{(0)}=H$.

And the output of second graph convolution layer is passed through a softmax layer to get distribution over all classes per node.

$$
Y=\operatorname{Softmax}\left(F^{(2)}\right)
$$

\subsection{Inductive Models}

Usage of transductive models is limited to certain test cases that have been seen by model during training. For an NLU system to support user queries, it is crucial to be able to generalize to unseen data. Thus, we use our proposed transductive models as an auxiliary model in an inductive semi-supervised learning pipeline, which is shown as Figure 2.

The input of pipeline is the combination of a few labeled utterances $X_{\text {train }}$ and a large amount 
of unlabeled data $X_{\text {unlabeled }}$ as mentioned in previous section. We apply paraphrase detection model on both $X_{\text {train }}$ and $X_{\text {unlabeled }}$ to find pairwise paraphrase relations between utterances. Details of the paraphrase detection model is given in Section 4.

For each utterance, we first find all its paraphrases as adjacency lists. We then build graph based on the adjacency lists. Transductive models (TeGrabS, TGCN, etc.) are applied as auxiliary model as shown in the graph, to predict labels for unlabeled data $X_{\text {unlabeled }}$ from both labeled data and graph structure. Finally, predictions of $X_{\text {unlabeled }}$ are fed into NLU model training set to re-train the NLU model and test on the unseen test set.

Based on the transductive TeGrabS and TGCN, here we propose their inductive variants, named Pseudo-Labeling with Text-Graph Beam Search (PL-TeGrabS) and Pseudo-Labeling with TextGCN (PL-TGCN) where TeGrabS and TGCN are used as auxiliary models in the aforementioned inductive semi-supervised learning pipeline, respectively.

\section{Paraphrase Detection for Graph Construction}

In this work, we leveraged paraphrase learning to find potential paraphrases in the data set and construct a graph. In real-world applications, this could be obtained from analyzing usage pattern, such as repetition or rephrase of user requests. In this work, we apply the paraphrase classification model on the NLU utterances to retrieve the paraphrase pairs within the data. We then construct the graph where paraphrases are connected.

In this section, we explain how the paraphrase model is trained as well as the construction of the graph.

\subsection{Paraphrase Embedding Learning}

In order to obtain embedding for paraphrases, we used a word averaging model. In this approach, once a word embedding matrix is learned, we average them over a sequence:

$$
g(x)=\frac{1}{n} \sum_{i}^{n} W_{w}^{x_{i}}
$$

where $W_{w}$ is a word embedding matrix. Parameters are learned by minimizing an objective func- tion with a margin, as described in Wieting et al. (2016a).

For embedding learning, we used the PPDB-S data set (Pavlick et al., 2015), which comprises 1.5 million paraphrase pairs.

\subsection{Paraphrase Classification}

Using the embeddings, we trained a model that outputs a score as an indicative for the pair to be paraphrases of each other. In the model, we used the embedding approach described in Section 4.1 and obtain an embedding $e$ for each utterance. For a pair of utterances $u_{1}$ and $u_{2}$, we combine their embeddings in the following way:

$$
h=\left[e_{u_{1}}, e_{u_{2}},\left|e_{u_{1}}-e_{u_{2}}\right|, e_{u_{1}} \times e_{u_{2}}\right]
$$

where we concatenate each utterance's embedding, element-wise difference and product between the two.

We then used a fully-connected network to output the probability for two utterances being paraphrases. We used two 100-dimension hidden layers with ReLU activation (Nair and Hinton, 2010) for the task. Further details of the embedding learning and classification model can be found in Cho et al. (2019b).

To train the paraphrase classification model, we used a back-translated paraphrase corpus (Wieting and Gimpel, 2017). For positive examples, we randomly selected $1.4 \mathrm{M}$ paraphrase pairs from the corpus. For negative examples, we randomly pair up utterances within the corpus so that the utterances in the pair are not paraphrases of each other. In the end, we obtained $2.8 \mathrm{M}$ pairs of data, with balanced positive and negative labels.

Using the method above leads to an F-score of 98.39 on a test set with balanced 20K pairs. Note that the performance of classification model is expected to regress when applied on the target task data, due to the domain mismatch between the sizable, publicly available paraphrase corpus (Wieting and Gimpel, 2017) and the NLU task data (Coucke et al., 2018).

In this work, we consider paraphrase pairs whose score returned by the model is higher than a threshold $\theta=0.99$. Detailed statistics on the constructed graph can be found in Section 6 .

\section{Experimental Setup}

In this section, we discuss the experimental setup used in this work. First, we describe the data sets 


\begin{tabular}{|l|l|}
\hline Intent & Utterances with slot labels \\
\hline searchFlight & find me a flight from [origin](Paris) to [destination](New York) \\
searchFlight & I need a flight leaving [date](this weekend) to [destination](Berlin) \\
searchFlight & show me flights to go to [destination](new york) leaving [date](this evening) \\
\hline
\end{tabular}

Table 1: Examples of the SNIPS Dataset.

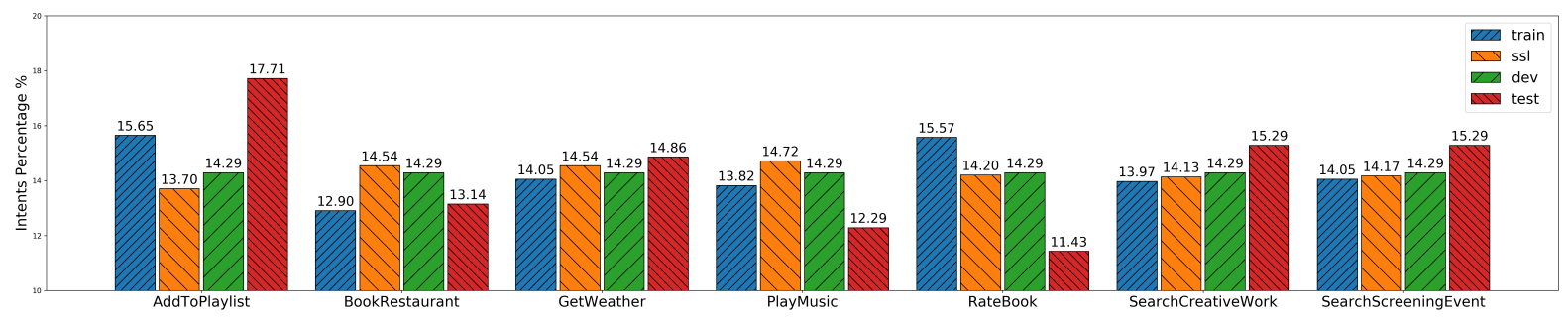

Figure 3: Intent distribution in SNIPS Train, SSL, Dev and Test sets.

\begin{tabular}{|l|r|}
\hline & No. Utterances \\
\hline Train & 1,310 \\
SSL candidate (unlablled) & 11,774 \\
Dev & 700 \\
Test & 700 \\
\hline
\end{tabular}

Table 2: SNIPS data statistics.

used for training and evaluating the suggested SSL approach. We also describe the NLU model used in this work, followed by description on the comparative systems.

\subsection{Data}

To evaluate the proposed model, experiments were performed on SNIPS dataset (Coucke et al., 2018), which is collected from the SNIPS personal voice assistant. This data comes with a pre-cut train, dev, and test sets, which contain 13,084, 700 and 700 utterances respectively. There are 72 slot labels and 7 intent types for the training set. Example utterances from the SNIPS data is shown in Table 1.

Designing a real-world application often faces with a challenge where there is an abundant amount of unlabeled data, but only a limited amount of labeled data. In order to simulate this scenario, we split the training data portion further, so that only $10 \%$ of the labeled training data is used for model training. The rest $90 \%$ of the labeled training data would be considered as candidates for SSL. Thus, we did not rely on the annotated labels in the SSL portion of the training data, but consider this as an unlabeled data and try to learn them from SSL process. Overall dataset statistics is given in Table 2, intent distributions in Train, SSL, Dev and Test set are shown in Figure 3.

\subsection{NLU System Description}

The NLU model we used is a Slot-Gated attentionbased bidirectional long short-term memory Model (SGM) (Goo et al., 2018). In our setting, the full-attention setup was used, which achieves the best performance in the paper. We used the default hyper-parameters from the code base. For TGCN and TeGrabS, graph structure was used to determine intent labels of unlabelled SSL candidates. Then, since Slot-Gated model leverages intent classification results to make slot predictions, graph SSL actually also helps in filling slots.

\subsection{Comparative systems}

In order to explore the effectiveness of the SSL approaches we discuss in this work, we rely on two comparative systems. First system ("Baseline") is trained only on training data in Table 2, without applying SSL. In the second system Pseudo Labelling Baseline ("PL-Baseline"), we applied SSL, more specifically, pseudo labelling, but without leveraging the graph structure. For this system, we first trained the Baseline then infer labels for unlabeled data $X_{\text {unlabeled }}$ with Baseline. After giving $X_{\text {unlabeled }}$ pseudo labels, we put it into NLU model training set, along with $X_{\text {train }}$, to retrain NLU model. 


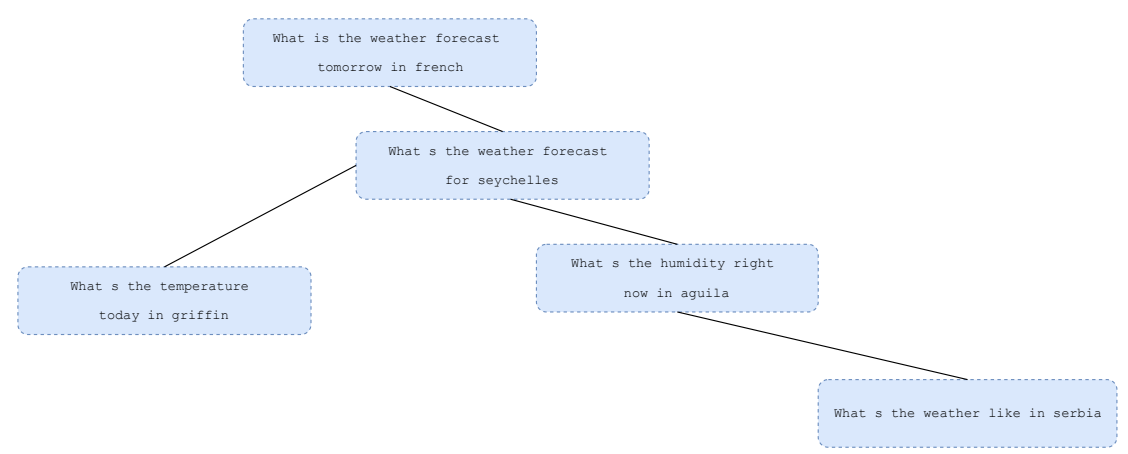

Figure 4: Excerpt of constructed graph using SNIPS data. Graph is constructed to represent utterance similarity using a paraphrase measure.

\begin{tabular}{lcccc}
\hline Model & IC Acc. & IC F1 & Slot F1 & SER \\
\hline Baseline* & 92.57 & $\mathbf{9 2 . 5 2}$ & 59.30 & 67.43 \\
PL-Baseline & 92.86 & 92.03 & 59.61 & 68.71 \\
PL-TGCN & $\mathbf{9 3 . 1 4}$ & 92.48 & $\mathbf{6 3 . 9 5}$ & $\mathbf{6 3 . 8 6}$ \\
PL-TeGrabS & 73.43 & 72.95 & 58.02 & 73.71 \\
\hline
\end{tabular}

Table 3: Transductive results on SNIPS data. *Baseline for Snips dataset is Slot Gated Modeling (Goo et al., 2018).

\subsection{Evaluation}

We report IC accuracy, F1 score, slot F1 and Slot Error Rate (SER) as metrics to measure the performance of the models.

SER is a metric used to combine intent classification accuracy and the slot classification accuracy in a single score. It is defined as:

$$
S E R=\frac{S+I+D}{S+D+C}
$$

where $\mathrm{S}$ is number of substitution errors for intents or slots, I is the number of insertion errors for intents or slots, $\mathrm{D}$ is the number of deletion errors for intents or slots, and $\mathrm{C}$ is the number of correct slots and intents.

\section{Results}

In this section, we first discuss the constructed graph using paraphrase measures. We then report the inductive SSL performance with graph methods as auxiliary models.

\subsection{Constructed Graph}

The paraphrase graph is built based on the train and SSL candidate set as we discussed in previous sections. Figure 4 is a part of the constructed graph, from which we can observe paraphrase patterns among connected components. We have also confirmed that neighbors in this excerpt share the same intent (GetWeather) as well as similar slots.

The whole graph contains 12,895 nodes (which indicates that there are duplicates in SNIPS dataset), 52,876 edges when we set the paraphrase threshold $\theta=0.99$.

\subsection{Inductive Results}

We evaluated baselines and our proposed models on the SNIPS dataset. Intent classification and slot filling experiment results are shown in Table 3.

We can observe that PL-TGCN outperformed other models on intent classification accuracy. However, this model is slightly defeated by baseline on intent classification F1-score. Our analysis revealed that PL-TGCN tends to predict more utterances into AddToPlaylist instead of PlayMusic, compared to baseline. Since AddToPlaylist is the biggest intent class in test set (124/700), more predictions in this class will certainly raise accuracy, but will do little harm to F1-score, given that we are reporting F1-score averaged from all classes. However, though Baseline did good job in not assigning more false positives to AddToPlaylist, it is more likely to assign utterances in AddToPlaylist to other classes, which is actually not good. Therefore, we can conclude that PL-TGCN achieved best performance on intent classification in general. It boosted the performance of slot filling through slot 
gate in SGM, leading to a great reduction on SER.

\section{Conclusion and Future Work}

In this work, we proposed transductive graphbased semi-supervised learning models as well as their inductive variants for NLU. In order to find similar utterances and construct a graph, we use a paraphrase detection model. To the best of our knowledge, our work is the first approach to apply text based graph structure for an SSL of NLU. We evaluate our method's applicability on publicly available data and model. Results show that applying the inductive graph-based semi-supervised learning can reduce the error rate of the NLU model by $5 \%$.

In the future, we will extend our work on other public datasets, explore methods to directly predict slots with transductive graph models. We will also make further research on applying other SSL techniques, e.g. iterative bootstrapping, instead of pseudo-labelling.

\section{References}

Mohammad Aliannejadi, Masoud Kiaeeha, Shahram Khadivi, and Saeed Shiry Ghidary. 2017. Graphbased semi-supervised conditional random fields for spoken language understanding using unaligned data. CoRR, abs/1701.08533.

Eunah Cho, He Xie, and William M Campbell. 2019a. Paraphrase generation for semi-supervised learning in nlu. In Proceedings of the Workshop on Methods for Optimizing and Evaluating Neural Language Generation, pages 45-54.

Eunah Cho, He Xie, John Lalor, Varun Kumar, and William M Campbell. 2019b. Efficient semisupervised learning for natural language understanding by optimizing diversity.

Alice Coucke, Alaa Saade, Adrien Ball, Théodore Bluche, Alexandre Caulier, David Leroy, Clément Doumouro, Thibault Gisselbrecht, Francesco Caltagirone, Thibaut Lavril, Maël Primet, and Joseph Dureau. 2018. Snips voice platform: an embedded spoken language understanding system for privateby-design voice interfaces. CoRR, abs/1805.10190.

Chih-Wen Goo, Guang Gao, Yun-Kai Hsu, Chih-Li Huo, Tsung-Chieh Chen, Keng-Wei Hsu, and YunNung Chen. 2018. Slot-gated modeling for joint slot filling and intent prediction. In Proceedings of the 2018 Conference of the North American Chapter of the Association for Computational Linguistics: $\mathrm{Hu}$ man Language Technologies, NAACL-HLT, New Orleans, Louisiana, USA, June 1-6, 2018, Volume 2 (Short Papers), pages 753-757.
William L. Hamilton, Zhitao Ying, and Jure Leskovec. 2017. Inductive representation learning on large graphs. In Advances in Neural Information Processing Systems 30: Annual Conference on Neural Information Processing Systems 2017, 4-9 December 2017, Long Beach, CA, USA, pages 1024-1034.

Thomas N. Kipf and Max Welling. 2016. Variational graph auto-encoders. CoRR, abs/1611.07308.

Thomas N. Kipf and Max Welling. 2017. Semisupervised classification with graph convolutional networks. In 5th International Conference on Learning Representations, ICLR 2017, Toulon, France, April 24-26, 2017, Conference Track Proceedings.

Samuli Laine and Timo Aila. 2017. Temporal ensembling for semi-supervised learning. In 5th International Conference on Learning Representations, ICLR 2017, Toulon, France, April 24-26, 2017, Conference Track Proceedings.

Ouyu Lan, Su Zhu, and Kai Yu. 2018. Semi-supervised training using adversarial multi-task learning for spoken language understanding. In 2018 IEEE International Conference on Acoustics, Speech and Signal Processing, ICASSP 2018, Calgary, AB, Canada, April 15-20, 2018, pages 6049-6053.

Bing Liu and Ian Lane. 2016. Attention-based recurrent neural network models for joint intent detection and slot filling. In Interspeech 2016, 17th Annual Conference of the International Speech Communication Association, San Francisco, CA, USA, September 8-12, 2016, pages 685-689.

Takeru Miyato, Shin-ichi Maeda, Masanori Koyama, and Shin Ishii. 2019. Virtual adversarial training: A regularization method for supervised and semisupervised learning. IEEE Trans. Pattern Anal. Mach. Intell., 41(8):1979-1993.

Vinod Nair and Geoffrey E Hinton. 2010. Rectified linear units improve restricted boltzmann machines. In Proceedings of the 27th international conference on machine learning (ICML-10), pages 807-814.

Shirui Pan, Ruiqi Hu, Guodong Long, Jing Jiang, Lina Yao, and Chengqi Zhang. 2018. Adversarially regularized graph autoencoder for graph embedding. In Proceedings of the Twenty-Seventh International Joint Conference on Artificial Intelligence, IJCAI 2018, July 13-19, 2018, Stockholm, Sweden., pages 2609-2615.

Shirui Pan, Jia Wu, Xingquan Zhu, Guodong Long, and Chengqi Zhang. 2017. Task sensitive feature exploration and learning for multitask graph classification. IEEE Trans. Cybernetics, 47(3):744-758.

Shirui Pan, Jia Wu, Xingquan Zhu, Chengqi Zhang, and Philip S. Yu. 2016. Joint structure feature exploration and regularization for multi-task graph classification. IEEE Trans. Knowl. Data Eng., 28(3):715728. 
Ellie Pavlick, Pushpendre Rastogi, Juri Ganitkevitch, Benjamin Van Durme, and Chris Callison-Burch. 2015. Ppdb 2.0: Better paraphrase ranking, finegrained entailment relations, word embeddings, and style classification. In Proceedings of the 53rd Annual Meeting of the Association for Computational Linguistics and the 7th International Joint Conference on Natural Language Processing (Volume 2: Short Papers), volume 2, pages 425-430.

Antti Tarvainen and Harri Valpola. 2017. Mean teachers are better role models: Weight-averaged consistency targets improve semi-supervised deep learning results. In Advances in Neural Information Processing Systems 30: Annual Conference on Neural Information Processing Systems 2017, 4-9 December 2017, Long Beach, CA, USA, pages 1195-1204.

Phi Vu Tran. 2018. Learning to make predictions on graphs with autoencoders. In 5th IEEE International Conference on Data Science and Advanced Analytics, DSAA 2018, Turin, Italy, October 1-3, 2018, pages 237-245.

Gokhan Tur and Renato De Mori. 2011. Spoken language understanding: Systems for extracting semantic information from speech. John Wiley \& Sons.

Petar Velickovic, Guillem Cucurull, Arantxa Casanova, Adriana Romero, Pietro Liò, and Yoshua Bengio. 2018. Graph attention networks. In 6th International Conference on Learning Representations, ICLR 2018, Vancouver, BC, Canada, April 30 - May 3, 2018, Conference Track Proceedings.

John Wieting, Mohit Bansal, Kevin Gimpel, and Karen Livescu. 2016a. Towards universal paraphrastic sentence embeddings. International Conference on Learning Representations (ICLR).

John Wieting and Kevin Gimpel. 2017. Paranmt-50m: Pushing the limits of paraphrastic sentence embeddings with millions of machine translations. arXiv preprint arXiv:1711.05732.

Zhang Xinyi and Lihui Chen. 2019. Capsule graph neural network. In 7th International Conference on Learning Representations, ICLR 2019, New Orleans, LA, USA, May 6-9, 2019.

Zhilin Yang, Junbo Jake Zhao, Bhuwan Dhingra, Kaiming He, William W. Cohen, Ruslan Salakhutdinov, and Yann LeCun. 2018. Glomo: Unsupervised learning of transferable relational graphs. In Advances in Neural Information Processing Systems 31: Annual Conference on Neural Information Processing Systems 2018, NeurIPS 2018, 3-8 December 2018, Montréal, Canada., pages 8964-8975.

Zhitao Ying, Jiaxuan You, Christopher Morris, Xiang Ren, William L. Hamilton, and Jure Leskovec. 2018. Hierarchical graph representation learning with differentiable pooling. In Advances in Neural Information Processing Systems 31: Annual Conference on Neural Information Processing Systems
2018, NeurIPS 2018, 3-8 December 2018, Montréal, Canada., pages 4805-4815.

Jiani Zhang, Xingjian Shi, Junyuan Xie, Hao Ma, Irwin King, and Dit-Yan Yeung. 2018a. Gaan: Gated attention networks for learning on large and spatiotemporal graphs. In Proceedings of the ThirtyFourth Conference on Uncertainty in Artificial Intelligence, UAI 2018, Monterey, California, USA, August 6-10, 2018, pages 339-349.

Muhan Zhang, Zhicheng Cui, Marion Neumann, and Yixin Chen. 2018b. An end-to-end deep learning architecture for graph classification. In Proceedings of the Thirty-Second AAAI Conference on Artificial Intelligence, (AAAI-18), the 30th innovative Applications of Artificial Intelligence (IAAI-18), and the 8th AAAI Symposium on Educational Advances in Artificial Intelligence (EAAI-18), New Orleans, Louisiana, USA, February 2-7, 2018, pages 44384445. 